\title{
Development and Test of a New Solar-Air Heat Exchanger for the Linear Mirror II System
}

\author{
Hans Grassmann ${ }^{1,2 *}$, Marco Citossi ${ }^{1}$ \\ ${ }^{1}$ Dipartimento Politecnico di Ingegneria e di Architettura (DPIA), University of Udine, Udine, Italy \\ ${ }^{2}$ Isomorph SRL, Trieste, Italy \\ Email: ^hans.grassmann@uniud.it, marco.citossi@uniud.it
}

How to cite this paper: Grassmann, $\mathrm{H}$. and Citossi, M. (2019) Development and Test of a New Solar-Air Heat Exchanger for the Linear Mirror II System. Smart Grid and Renewable Energy, 10, 155-164. https://doi.org/10.4236/sgre.2019.105010

Received: April 15, 2019

Accepted: May 27, 2019

Published: May 30, 2019

Copyright ( 2019 by author(s) and Scientific Research Publishing Inc. This work is licensed under the Creative Commons Attribution International License (CC BY 4.0).

http://creativecommons.org/licenses/by/4.0/

\begin{abstract}
The Linear Mirror II is an innovative system to concentrate solar energy, developed by Isomorph SRL. In this paper, a solar-air heat exchanger of new conception is presented and tested together with a Linear Mirror II. The heat exchanger surface is selective with respect to direction and position of light absorption and emission and once heated by the Linear Mirror II, can reach an air temperature of up to $230^{\circ} \mathrm{C}$.
\end{abstract}

\section{Keywords}

Renewable Energy, Solar Biomass Conversion, Concentrated Solar Power, Solar Carbon, Solar Pyrolysis, Linear Mirror, Heat Exchanger

\section{Introduction}

Already in many countries, a relevant fraction of electric energy is provided by renewable energy sources, mostly photovoltaic, wind energy and hydro energy [1] [2]. In comparison, the contribution of renewable energy sources to thermal energy consumption is much lower [3], in spite of the fact that industry as well as private homes consumes more thermal energy than electric energy [4]. As a consequence, new and improved systems for providing renewable heat energy become a necessary condition for further reducing the level of $\mathrm{CO}_{2}$ production [5] [6].

These new technologies should provide thermal energy at high temperatures also in winter, and even at northern latitudes, in a simple and economic way [6] [7]. The Linear Mirror technology was developed to meet these requirements [8].

It consists of a matrix of eight mirrors, each composed of four segments, which are slightly inclined with respect to each other. The mirrors are connected 
by steal levers in such a way that moving concurrently, they reflect the sun light onto a fixed spot during the day, thereby concentrating the sun light. The result is a very cheap, simple and scalable system.

In its standard version, the Linear Mirror provides hot water at temperatures up to $100^{\circ} \mathrm{C}$ also in winter, and with a rather high efficiency, due to the low specific heat of its absorber [9]. The temperature limit of $100^{\circ} \mathrm{C}$ is applied by a control software in order to simplify the integration of the Linear Mirror into existing heating systems.

The Linear Mirror can also be used to provide higher temperatures: in an experiment which produced solar carbon from simple biomasses and only using solar energy, a temperature of up to $500^{\circ} \mathrm{C}$ was reached [10].

In order to use water as a heat carrier at temperatures above $100^{\circ} \mathrm{C}$, high pressure installations are needed [11]. This can make the whole setup complex and expensive and may create safety problems. In addition, transferring the heat from the pressurized water to the application process might be difficult and inefficient. For instance, it would be difficult to torrify or pyrolyze cheap biomasses by means of hot water, since one should use rotating drums or similar devices, or additional water/air heat exchangers [12].

It would be simpler instead, if a flow of hot air would be available. Then cheap biomasses could be roasted in the same way as coffee is roasted, with a simple and economic procedure. Unfortunately, the hot air which is needed to roast coffee is provided by a gas flame, which makes the whole process not $\mathrm{CO}_{2}$ neutral. It would be of great advantage to have a heat exchanger available, which can produce hot air just using concentrated sun light.

\section{Requirements for a High Temperature Solar Heat Exchanger Working with Air}

An important element of solar heat exchangers is their selective absorbing surface. The heat exchanger must be capable to efficiently absorb sun light well instead of reflecting it, and must emit as little thermal radiation as possible. It should consequently have a high absorptivity for sun light and a low emissivity for thermal radiation [13].

According to Kirchhoff's law of thermal radiation, the absorptivity and the emissivity of a surface are equal, but not in a global sense, rather they will be functions of the wavelength of the electromagnetic radiation and also of the direction of the photons [14].

Selective absorbing surfaces, used in solar systems make use of this fact: they have a high absorptivity (and emissivity) at the wavelength of the incident sun light, and a small emissivity (and absorptivity) at the much larger wavelength of the thermal radiation, emitted by the surface.

For example, the standard absorber used for heating water with the Linear Mirror (made by the company Energie Solaire (Switzerland)), is equipped with this kind of surface. Unfortunately, its selective surface would not withstand 
high temperatures, and therefore cannot be used to heat air to high temperatures.

This leads, first, to the question, whether selectivity can be provided to an adsorber to a different way than using a wavelength-dependent absorptivity. A second requirement comes from the fact that the coefficient of heat transmission is very low for air, therefore, the solar heat exchanger uses air. In the next chapter we describe, how these two requirements-first a selective surface, second a large surface-can be combined into a very simple solution.

\section{Basic Properties of the Isomorph Solar-Air Heat Exchanger}

Our solar heat absorber is made of stainless-steel plates, arranged in a bellow shaped structure, as shown in Figure 1, so that incident light rays hitting the structure undergo multiple reflections. In each reflection the light ray loses part of its energy, heating the surface.

Let's assume that, a light ray-as shown in Figure 1-enters parallel to the horizontal $x$-axis. If the opening angle between the plates is $10^{\circ}$, and if one assumes an absorptivity $a=0.2$, the light ray will be deflected away from its original direction by a $10^{\circ}$ angle at each reflection. It will therefore undergo 18 $\left(180^{\circ} / 10^{\circ}\right)$ reflections and travel back along the horizontal direction: its intensity will then be reduced by a factor of $(1-a)^{18}=0.02$.

More generally, one can relate the incident light intensity $I_{i n c}$ with the reflected intensity $I_{\text {ref }}$ (after $n$ reflections) by means of the "effective" reflectivity $r_{\text {eff }}$ such that: $I_{\text {ref }}=I_{\text {inc }} \cdot r_{\text {eff }}$, and $r_{\text {eff }}$ can be expressed in terms of the characteristic surface reflection factor $r: r_{\text {eff }}=r^{n}$. Similarly, one can define an "effective" absorptivity $a_{\text {eff }}$ as: $a_{\text {eff }}=1-r^{n}$.

One can then compare the intensity emitted by the heat radiation into the very direction of the previous incident light ray at the same spatial position. This is shown in Figure 2, where the heat radiation emitted along the direction of the incident light of Figure 1 is shown.

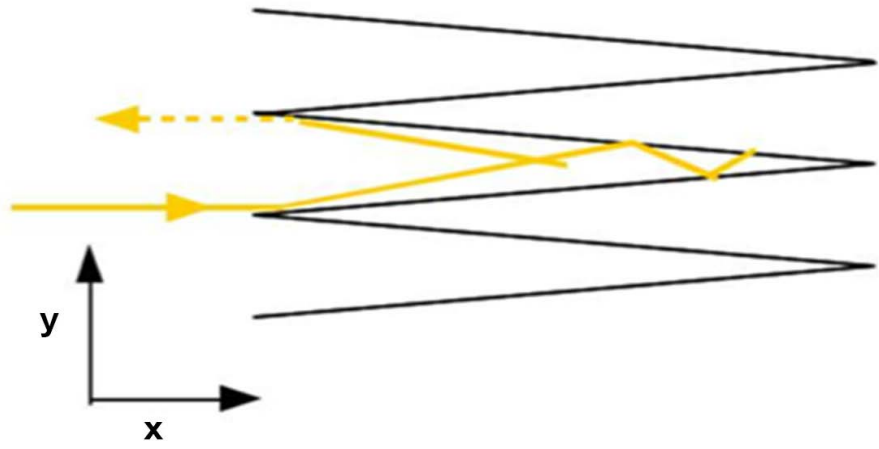

Figure 1. An incident light ray (yellow, solid line) undergoes multiple reflections on a metallic surface, until it finally bounces back (dotted line), each reflection reduces the light intensity. Not all reflections are shown in the figure. 


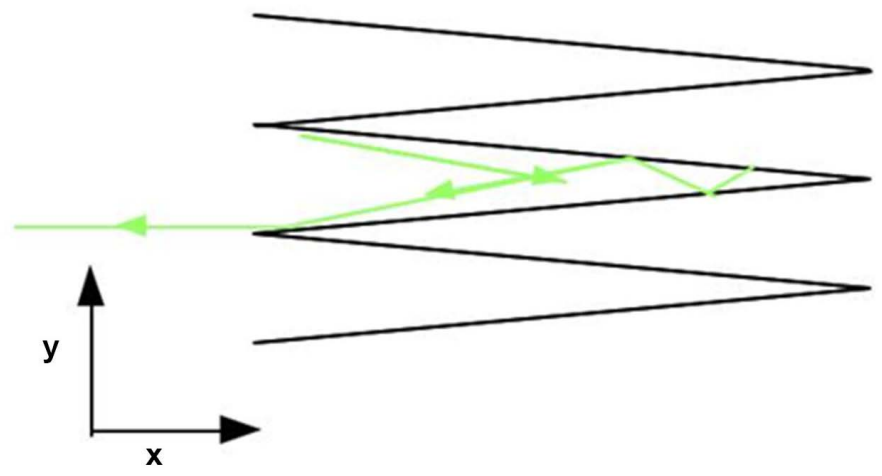

Figure 2. Radiation emitted along the direction of the incident sun light shown in Figure 1, at the same position. An observer in this direction will see not only the light emitted directly from a surface element, but also the reflection of light rays emitted from other surface elements in other positions, as indicated in this figure (as in Figure 1, not all ray elements are shown).

The thermal radiation emitted in this direction has several components. There is the direct thermal radiation emitted from a given surface element, but in addition there is the heat radiation which impinges on that surface from other surfaces and is then reflected along this very same direction at the same position. The geometric case is identical between Figure 1 and in Figure 2.

The radiation emitted from a single surface element, without having had any previous reflection, has intensity $I_{0}=I_{b} \cdot e$, where $I_{b}$ is the black body thermal radiation intensity and $e$ is the emissivity factor. The thermal radiation intensity which comes out from that surface element, after having undergone before $k$ reflections will be instead $I_{n}=I_{b} \cdot e \cdot r^{k}$. This gives a total emitted intensity from that particular surface element of $I_{t o t}=\sum_{i=0}^{k} I_{b} \cdot e \cdot r^{i} \quad$ (the geometric series). The ratio between the total emitted intensity and the black body radiation intensity is the effective emissivity $e_{\text {eff. }}$. By definition $e_{e f f}=\frac{I_{t o t}}{I_{b}}$ and so the evaluation of the geometric series gives $e_{\text {eff }}=\frac{e \cdot\left(1-r^{k+1}\right)}{1-r}=1-r^{k+1}$.

When comparing the two expressions $e_{e f f}=1-r^{k+1}$ and $a_{e f f}=1-r^{n}$ one needs to take into account, that by its very nature the geometric series has a particular counting convention, as is shown in Figure 3 with an example.

In Figure 3 on the left sketch the incident light is reflected twice, so that $n=2$. On the right sketch, instead, the emitted light is described by $k=1$, so that $k+1$ $=2$ which is equal to $n$, for $k+1=n$.

It turns out then, that the effective absorptivity, $a_{\text {eff }}$, and the effective emissivity, $e_{\text {eff }}$ are equal, but can much differ from the material properties, $a$ and $e$, of the metallic surface.

It is now clear (and it can also be seen for example by comparing Figure 1 and Figure 2 with Figure 3), that the number of reflections respectively depends on the angle of incidence or emission. 

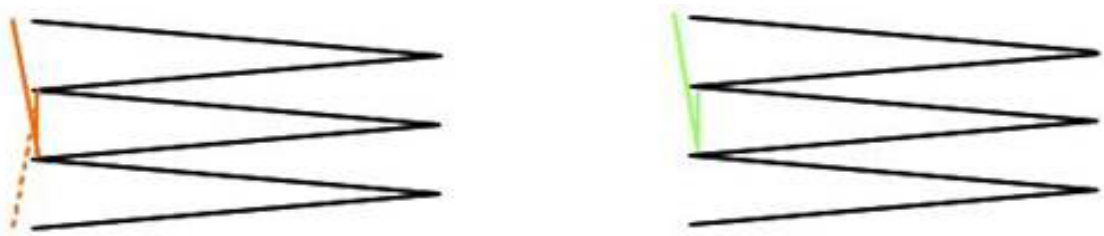

Figure 3. Left: an incident light ray impinging at a large angle indicated by a solid line is reflected twice before being emitted from the absorber (dotted line). Right: a thermal radiation ray emitted with the same spatial and directional parameters as the incident light ray (shown in the left part of the figure), but travelling in the opposite direction.

This means that also, $a_{\text {eff }}$ and $e_{\text {eff }}$ depend on the angle with respect to the $x$-axis. radiation absorbed or emitted at a large angle will have a small associated absorptivity or emissivity, when compared to radiation which enters in the bellow-shaped structure with a direction close to the $x$-axis.

The concentrated light from the Linear Mirror incident on the heat exchanger has a direction close to the horizontal ( $x$-axis in Figure 1 ). Therefore, it will be absorbed very well, though the surface of the absorber consists of reflecting stainless steel. The thermal radiation emitted from the hot surface instead will be emitted into all directions and in average it will be emitted with a low emissivity.

This new heat exchanger has therefore selective properties: it absorbs well the sun light and at the same time emits little thermal radiation. The process depends on the directionality, rather than on the wavelength.

There is a second effect, which presumably can increase the performance of such a bellow-shaped absorber: for a given direction of incidence, the number of reflections of a light ray will always be the same, regardless of where it first hits the surface. The density of these reflections instead will very much depend on the initial point of incidence. Rays incident close to each bellow vertex will deposit most of their energy on a very small surface, heating then the steel very much. This means that even if the incident radiation is distributed uniformly along the y-direction, the surface will not show a uniform temperature at all: it will be hottest in a region, which supposedly will be relatively well protected against heat loss to the environment. A detailed study of this effect is however not the priority oh this article and should be studied in a further dedicated investigation.

All these discussions are based on a first approximation and for a precise evaluation of all the effects involved in the described process, a computer simulation would be needed, for instance to take into account the fact that not all of the light will be reflected since a certain fraction of the light will be diffused as well.

\section{Design and Test of the Solar-Air Heat Exchanger}

The new heat exchanger has been recently built and tested using the concentrated solar light provided by the Linear Mirror.

A drawing of the device is shown in Figure 4: behind the metallic bellow structure there is a flow of air which is produced by a ventilator of variable speed 


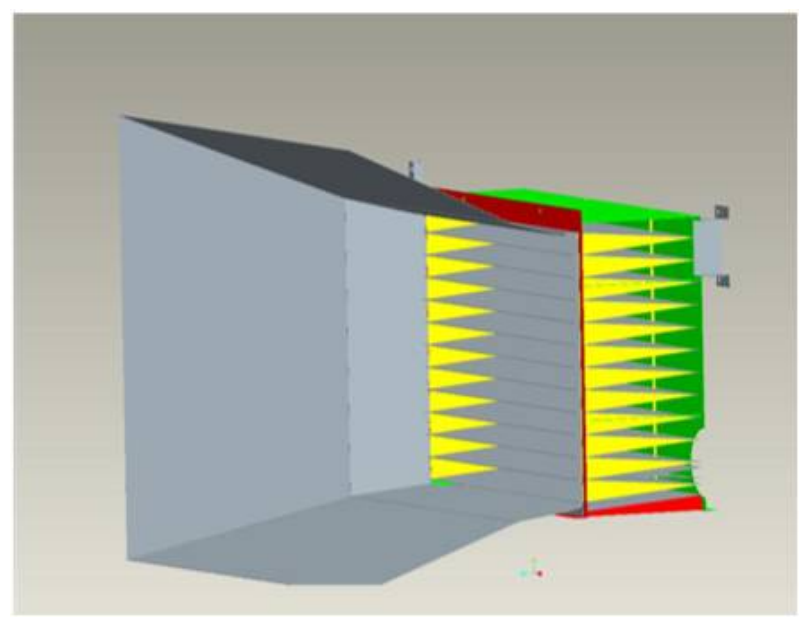

Figure 4. Side view of the solar-air heat exchanger. The bellow structure is in contact with a flow or air. A secondary mirror directs stray light onto the absorber surface and protects the absorber surface from the presence of atmospheric wind.

and heated by the hot metal surface. The heat exchanger can be operated as part of a closed circuit, for instance it could be operated under pressure, in future versions of the device.

The bellow structured heat echanger-which is receiving the concentrated light from the Linear Mirror-is $70 \mathrm{~cm}$ wide, $54 \mathrm{~cm}$ high and $25 \mathrm{~cm}$ thick. It is made of stainless steel and the center of the device is at about $1 \mathrm{~m}$ from the ground. The thickness of its wall is $1 \mathrm{~mm}$. The heat exchanger was positioned at a distance of $5 \mathrm{~m}$ from a Linear Mirror II. The bellow structure was surrounded by a secondary mirror, which protected the heat exchanger against wind as well. The Linear Mirror II presented an aperture surface of $13.8 \mathrm{~m}^{2}$ and its mean concentration factor on the heat exchanger was 13.8/0.7-0.54 $=36$.

\section{Test Results}

The air-heat exchanger presented here, has been developed and built based on general theoretical ideas, not from a detailed thermodynamic simulation. Its performance therefore is not optimized. Indeed, the purpose of this study is not to produce a detailed analysis of the performances: rather, to verify whether such a device of new concept can work in a way, which is useful for practical applications.

In Figure 6, part of the solar-air heat exchanger (shown previously in Figure 5) is covered, for the sake of comparison, with the solar water-heat exchanger (produced by Energie Solaire), which is normally used with the Linear Mirror for heating water ${ }^{1}$.

Figure 6 confirms that, as expected, the new solar-air heat exchanger reflects only a small fraction of the incident concentrated sun light. With respect to the

${ }^{1}$ In Figure 6 the secondary mirror has been removed. 


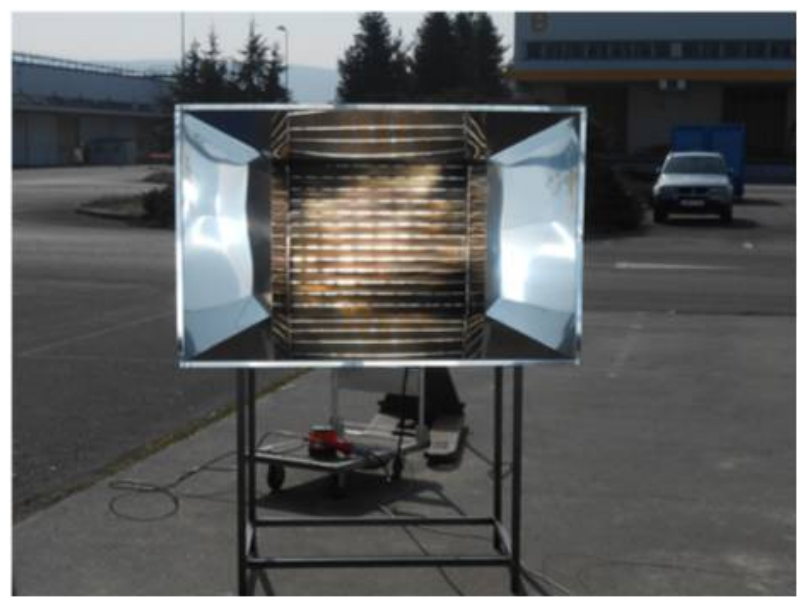

Figure 5. The solar-air heat exchanger illuminated by the concentrated sun light from the Linear Mirror II system.

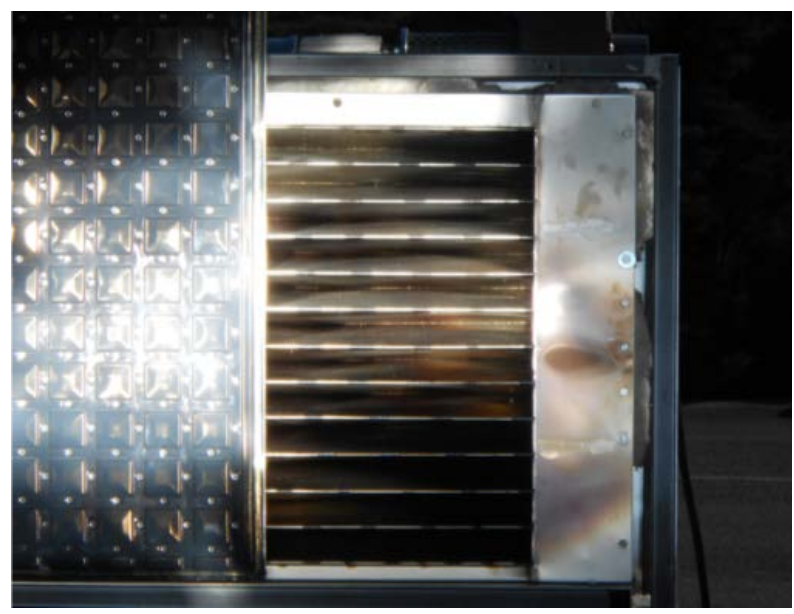

Figure 6. Comparison between a conventional heat exchanger with wave length selectivity (on the left) and the newly developed heat exchanger (on the right).

other, traditional heat exchanger. Note, that the Energie Solaire heat exchanger is a high quality product, with one of the best absorptivity values available on the market.

The new air-heat exchanger was exposed to the concentrated sun light on a sunny day in March. A ventilator was pushing a flow of ambient air through the heat exchanger and the flow was measured using a hot wire anemometer. Both the temperatures of the ambient and hot air, exiting the heat exchanger, were measured. From the difference between these two temperatures and the value of the air flow, the heating power of the device was calculated.

The thermal power as a function of the air temperature is reported in Figure 7 , which shows that the power provided by the solar-air heat exchanger diminishes by only about $10 \%$ going from $100^{\circ} \mathrm{C}$ to $230^{\circ} \mathrm{C}$ This indeed makes this kind of solar-air heat exchanger a useful device for applications up to that high values temperature. 


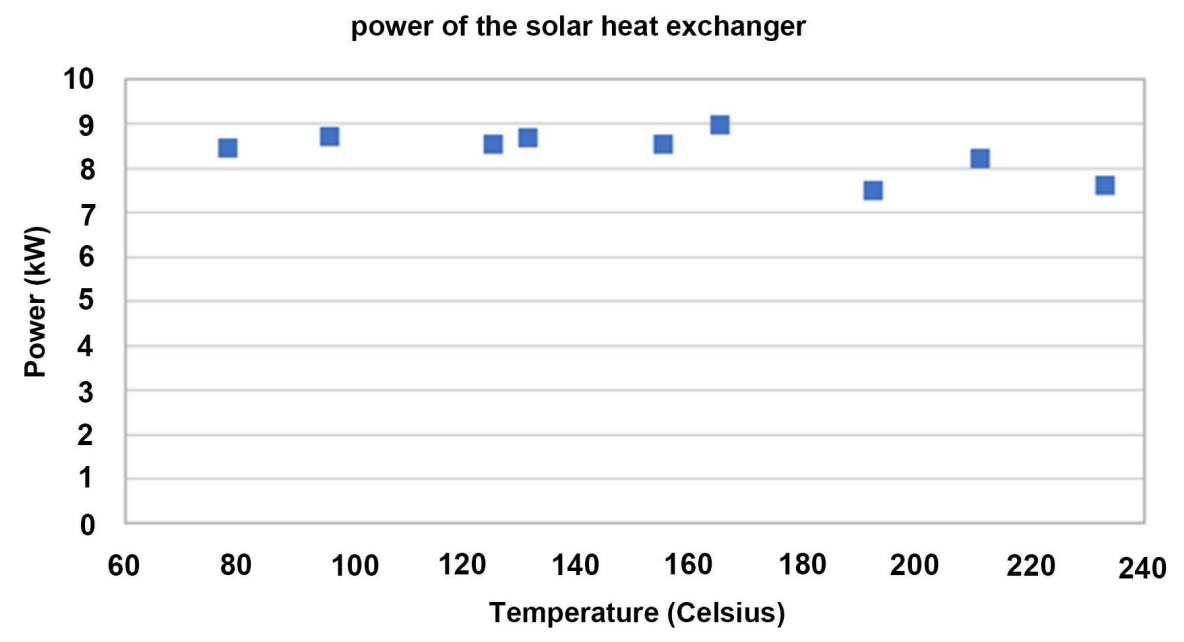

Figure 7. Thermal power of the solar heat exchanger operated with the Linear Mirror II system.

In addition, the quantity of heat energy harvested with this device is quite remarkable. For instance, at a temperature of $80^{\circ} \mathrm{C}$ it provides almost $9 \mathrm{~kW}$ on a sunny day, which is about as much as a conventional solar water heat exchanger provides at this temperature.

\section{The Linear Mirror as an IoT Element}

Hot air from solar energy can be used for many different applications, not only for heating buildings or providing energy to industrial processes. Torrefaction of biomasses, solar cooling, and for instance production of pressurized steam for electricity generation can be other applications.

In particular, one can note that the efficiency of the solar-air heat exchanger, as evidenced in Figure 7, can be still increased by means of a higher solar concentration factor. The Linear Mirror is made in a modular way. Additional mirrors could thus be installed, which increase the light concentration. In that case, temperatures well above $230^{\circ} \mathrm{C}$ might also be possible, being limited only by the optical and mechanical properties of steel.

The Linear Mirror with its solar-air heat exchanger could then be used for dedicated purposes at different times, depending from the actual consumer needs. For instance this device could be used in winter for heating, in summer for cooling and at other times for providing process heat.

In practice, this versatility can be achieved only if the Linear Mirror is able to communicate via internet, which means is a part of a smart grid as an element of the Internet of things (IoT).

We have therefore created a version of the control software, which runs on a Siemens Logo! 24 CE PLC, and which includes a solar tracker.

Since the logic module can operate as a web server, the Linear Mirror can be used as an element of the IoT, interacting with other components, receiving for instance meteorological information from internet or other messages. 


\section{Conclusions}

Isomorph developed a new solar-air heat exchanger, characterized by an absorber surface which is selective with respect to the direction of the incident and emitted radiation, while conventional heat exchangers are selective with respect to the wavelength. This heat exchanger has been tested and the results obtained show that it works about as well as the conventional solar water-heat exchanger (with a wavelength selective surface) previously with the Linear Mirror setup. Furthermore, it allows to reach much higher temperatures: in our first test, $230^{\circ} \mathrm{C}$ was reached without problems.

The solar-air heat exchanger was designed and built starting from fundamental general physics considerations, not from a detailed computer simulation. It can then be expected that further improvements will be reached in the future. Already now this new solar-air heat exchanger, together with the Linear Mirror solar concentrator, opens up many new possible applications for the solar thermal energy.

\section{Acknowledgements}

This work is part of a research program focused on the application of the solar-air heat exchangers to the waste sector. This project has been launched by $\mathrm{L}$. Aita, R. Bernes and A. Piani from A\&T 2000 SpA (http://www.aet2000.it), and would not have been possible without their valuable support.

\section{Conflicts of Interest}

The authors declare no conflicts of interest regarding the publication of this paper.

\section{References}

[1] Ellabban, O., Abu-Rub, H. and Blaabjerg, F. (2014) Renewable Energy Resources: Current Status, Future Prospects and Their Enabling Technology. Renewable and Sustainable Energy Reviews, 39, 748-764. https://doi.org/10.1016/j.rser.2014.07.113

[2] The Outlook for Energy: A View of 2040, Exxon. http://cdn.exxonmobil.com

[3] Jackson, D.Z., Stillerman, K.P. and Montgomery, D.R. (2016) Renewable Energy Can Provide $80 \%$ of U.S. Electricity by 2050 . Science for a Healthy Planet and Safer World. http://www.ucsusa.org/clean-energy/increase-renewable-energy

[4] Twidell, J. and Weir, T. (2005) Renewable Energy Sources. 2nd Edition, Taylor \& Francis, London. https://doi.org/10.4324/9780203989302

[5] Eke, M. (2017) The Advantages of Renewable Energy. https://guardian.ng/opinion/the-advantages-of-renewable-energy

[6] Renewables 2018 Global Status Report. REN21 (2018) Steering Committee. http://www.ren21.net/gsr-2018/

[7] Zhang, H.L., Baeyens, J., Degrees, J. and Caceres, G. (2013) Concentrated Solar Power Plants: Review and Design Methodology. Renewable and Sustainable Energy Reviews, 22, 466-481. https://doi.org/10.1016/j.rser.2013.01.032

[8] Grassmann, H., et al. (2013) First Measurements with a Linear Mirror Device of 
Second Generation. Smart Grid and Renewable Energy, 4, 253-258.

https://doi.org/10.4236/sgre.2013.43030

[9] http://www.isomorph-production.it/docs/TRIsomorph-rev-2.pdf

[10] Grassmann, H., Boaro, M., Citossi, M., Cobal, M., Ersettis, E., Kapllaj, E. and Pizzariello, A. (2015) Solar Biomass Pyrolysis with the Linear Mirror II. Smart Grid and Renewable Energy, 6, 179-186. https://doi.org/10.4236/sgre.2015.67016

[11] Duffie, J. and Beckmann, W. (2006) Solar Engineering of Thermal Processes. 3rd Edition, John Wiley and Sons, New Jersey.

[12] Basu, P. (2013) Biomass Gasification, Pyrolysis and Torrefaction. 2nd Edition, Academic Press, Boston. https://doi.org/10.1016/B978-0-12-396488-5.00004-6

[13] Montes, M.J., Abánades, A. and Martínez-Val, J.M. (2009) Performance of a Direct Steam Generation Solar Thermal Power Plant for Electricity Production as a Function of the Solar Multiple. Solar Energy, 83, 679-689. https://doi.org/10.1016/j.solener.2008.10.015

[14] Baltes, H.P. (1976) On the Validity of Kirchhoff'S Law of Heat Radiation for a Body in a Nonequilibrium Environment. 